\title{
LA PENSÉE SYSTÉMIQUE DES TRANSITIONS APPLIQUÉE À L'EST DE LA RDC UN MODÈLE DE LA RÉSISTANCE A LA RÉSILIENCE
}

\author{
Author(s) / Auteur(s) : \\ Rodrigue IYEMBO NGINDA \\ Licencié en Informatique de Gestion \\ Assistant MIS (Management Information System) du \\ Professeur Alphonse Christian IVINZA LEPAPA (Membre S\&O) \\ rodriveille@gmail.com
}

\begin{abstract}
Résumé :
Cet article introduit le concept de la résilience dans la pensée systémique des transitions contextualisé par rapport à la situation sociale que vivent les populations de l'Est de la RD Congo ; La notion de résilience correspond à la capacité d'un système à intégrer une perturbation dans son fonctionnement, sans changer de structure qualitative. L'un de ses intérêts est qu'elle relativise le caractère positif d'un certain nombre de concepts systémiques, tel que la stabilité et l'équilibre. Un système sera dit résilient lorsqu'il est capable de se maintenir alors qu'il est affecté par des perturbations.

$L$ 'Est de la RD Congo présente une instabilité grandissante de risques, et une grande incertitude sur le futur. Les populations sont frappées par des chocs multiples, comme les conflits armés systémiques, les violences interethniques, l'épidémie à virus Ebola ou les viols liés au genre, qui ont un impact direct sur les moyens d'existence des populations. (UNICEF, OECD 2014)

Comment envisager l'intervention systémique des transitions en incluant le courant de pensée de la résilience au sein des populations de l'Est de la RD Congo?

Le social est devenu intenable pour la majorité des Congolais. Il l'est surtout pour la jeunesse en ce moment où les fondamentaux de l'économie s'effondrent. Il s'est particulièrement aggravé ces deux dernières décennies suite aux guerres répétitives avec son lot des millions de morts des civils et la misère dans laquelle baigne l'écrasante majorité de la population. Il faut une réflexion méthodique, ordonnée et approfondie de la nature de l'être congolais. (MUTINGA, 2001, p.13)

Cet état d'instabilité est nourri, entre autres, par des vagues successives des crises humanitaires chroniques, des violations graves des droits de l'Homme, l'exploitation illégale des ressources naturelles, l'ingérence de la communauté internationale, l'impunité décriée par tous et de tout temps, et la faiblesse des systèmes judiciaire et pénitentiaire. (JACQUEMOT, 2009, p.83)

L'analyse systémique des transitions que nous allons appliquer à l'Est de la RD Congo utilisera les concepts de risque, de résilience et approche des moyens d'existence durables pour les communautés.
\end{abstract}

Keywords / Mots-clés :

risque, résilience, approche systémique, transition, système, résistance

\section{INTRODUCTION}

Le but de cet article est de proposer une réflexion qui va se pencher sur une analyse systémique de la résilience à l'Est de la République Démocratique du Congo ; Ensuite, nous aborderons également les concepts de transition systémique et enfin proposer un modèle systémique de la résilience.

L'Est du Congo présente un panorama complexe et changeant de risques, et une grande incertitude sur le futur. Les populations sont frappées par des chocs multiples et de différente intensité, comme les conflits armés, les viols à répétition, qui ont un impact sur les moyens d'existence des foyers; Et tous ces problèmes sociaux nous permettra de parler de la résilience (UNICEF, OECD, 2014).

La résilience offre aux gens la possibilité de prendre de meilleures décisions face aux risques auxquels ils sont confrontés. On entend souvent que la résilience permet de rapprocher les acteurs humanitaires et de développement pour un but commun; Elle serait le maillon manquant entre l'urgence et le 
développement, entre la réduction des risques de catastrophes et la prévention des conflits. Il n'y a pas de guide technique expliquant clairement comment « faire » des programmes de résilience.

\section{LE CONCEPT DE RÉSILIENCE}

Le terme même de résilience vient du latin Resilio qui signifie rebondir, ainsi est résilient l'objet capable de résister et de trouver un nouveau souffle après un choc; La résilience est une notion utilisée à l'origine en physique pour désigner la résistance d'un matériau au choc.

En écologie, elle prend un sens différent : dans son acception traditionnelle, elle mesure le temps de retour à l'équilibre d'un système après une perturbation. Depuis, sa définition a beaucoup changé ; Celle qui est communément admise aujourd'hui est formulée par (Holling, 1973). Il définit la résilience comme la capacité d'un système à pouvoir intégrer dans son fonctionnement une perturbation, sans pour autant changer de structure qualitative.

En management, on parlera de résilience stratégique qui n'est pas une manière de répondre à une crise passée ; Mais plutôt la capacité d'anticiper le changement avant que la nécessité de changer devienne une évidence. Elle consiste à anticiper et à ajuster continuellement des tendances qui peuvent en permanence détériorer la capacité cœur de métier à générer des revenus (Ivinza Lepapa, 2019, p.20).

La capacité d'un système ouvert à se maintenir lorsqu'il est affecté par une perturbation, et donc à se reproduire, se définit comme sa résilience, qui est une propriété systémique. La définition traditionnelle de la résilience en écologie repose sur l'idée d'un système en équilibre stable dont le comportement est prévisible et, dans cette acception, la résilience devient équivalente à la notion de stabilité d'un système autour d'un point d'équilibre (Holling, 1986).

La figure 1 nous illustre qu'un système en équilibre stable revient rapidement à l'équilibre, lorsqu'il s'en est écarté. Un état d'équilibre est dit stable si, après une petite variation des conditions initiales, le système retourne à cet état (point $\mathrm{B}$ ). Si cette petite perturbation entraîne, au contraire, le système irrémédiablement loin de son équilibre, celui-ci sera qualifié d'instable (point A).

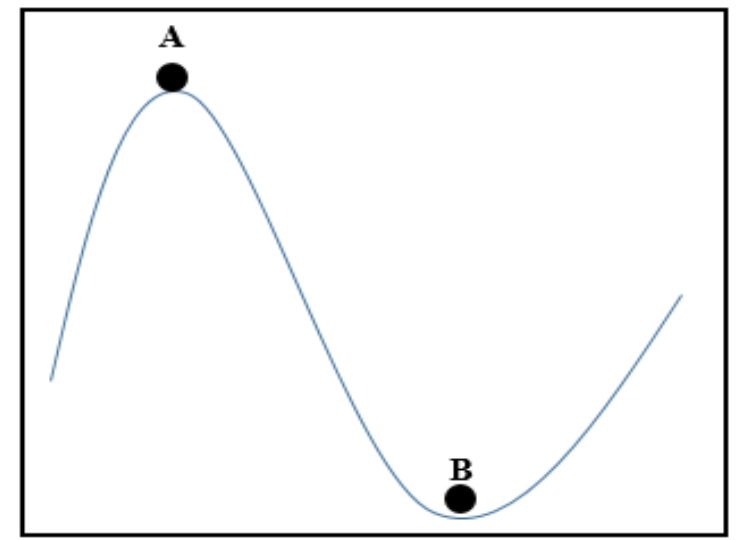

Figure 1. : Notion d'équilibre. Équilibre Instable (A) : une perturbation entraine le système immédiatement loin de son point d'équilibre. Équilibre Stable (B) : le système résiste à sa propre mise en équilibre. Il revient rapidement à l'équilibre après s'en être écarté.

\section{Les facteurs de la résilience}

Il n'existe pas encore de véritable théorie formalisée pour expliquer la résilience. Cependant, de nombreuses études soulignent l'importance de quelques facteurs déterminants. Parmi les facteurs positifs qui augmentent la résilience d'un système soumis à une perturbation, trois sont souvent cités :

- la diversité ;

- l'auto organisation ;

- l'apprentissage. 
En écologie, la perte de biodiversité est considérée comme un facteur qui réduit la résilience de l'écosystème. En outre, la résilience systémique est directement proportionnelle à l'auto organisation du système. Les colonies d'insectes, de fourmis ou d'abeilles, sont de bons exemples de systèmes auto organisés. Peu fragiles, ils ont une grande capacité à se restaurer, car les fonctions des "parties » endommagées sont prises en charge par les autres éléments. Les systèmes auto organisés sont donc très résilients.

Enfin, la résilience dépend de la capacité d'un système à s'adapter, ce qui est le cas des sociétés humaines grâce à l'apprentissage. Par exemple, dans une société où la population est bien préparée à réagir face à un type d'événement catastrophique des comportements de panique sont moins à craindre qu'au sein d'une population mal informée. Ainsi, au Japon, les scènes de panique sont exceptionnelles lors des séismes, alors qu'elles sont fréquentes en Turquie ou en Italie. Cette remarque n'implique pas que toute la chaîne de prévention des risques soit optimale au Japon. En sens inverse, divers facteurs réduisent la résilience sociale. De même ce comportement peut être applicable à la population vivant à l'Est de la RD Congo.

\section{LE CONCEPT DE TRANSITION SYSTÉMIQUE}

La notion de transition reste floue et peut recouvrir plusieurs visions selon les auteurs ou les décideurs. Il existe un concept scientifique de la transition quand on parle de systèmes. Elle peut être entendue comme le «processus de transformation au cours duquel un système passe d'un régime d'équilibre dynamique à un autre régime » (Bourg \& Papaux, 2015). Il s'agit là d'un modèle générique qui a donné lieu par exemple à la notion de transition démographique (Chabot, 2015).

Il existe deux grandes approches de la transition que l'on pourrait qualifié de complémentaires.

L'approche de la transition la mieux connue est celle de Rob Hopkins qui a rendu le terme populaire à la fin des années 2000 ; Sa caractéristique est de se baser sur l'initiative citoyenne et le développement local. La seconde approche est celle de l'action publique qui est politique et descendante. C'est celle de la transition écologique et solidaire ou de la transition énergétique (Theys, 2017) ; Elle englobe l'environnement et le développement économique. Elle peut parfois inclure la sphère sociale, la culture et l'éducation.

\section{La théorie des systèmes}

La théorie des systèmes (cybernétique) (Walliser, 1977) ou la théorie des catastrophes peuvent servir de guide pour appréhender des phénomènes transdisciplinaires. La théorie des systèmes cherche à rendre compte des fonctionnements et des dysfonctionnements des systèmes, générateurs de crises et de risques propose ainsi des méthodes d'analyse des risques technologiques construites sur la théorie des systèmes.

La cindynique (Yacov, 1998) est largement fondée sur l'étude des systèmes complexes dont la complexité est accentuée par la multiplicité des interactions qui unissent les différentes composantes d'une catastrophe.

\section{ANALYSE SYSTÉMIQUE DE LA RÉSILIENCE À L'EST DE LA RD CONGO}

Pour renforcer la résilience, il faut tout d'abord analyser comment un système a réagi aux chocs dans le passé, en s'appuyant sur un panorama des risques liés à un contexte donné.

L'analyse systémique de la résilience :

- Englobe non seulement les risques majeurs, mais aussi les séries de petits chocs dont l'impact sur les moyens d'existence des populations peut être tout aussi dévastateur.

- Etudie comment les facteurs de stress à long terme peuvent influencer la nature et l'impact des chocs à l'avenir.

- Sélectionne les composants du système des moyens d'existence par lesquels se propage l'impact négatif des chocs, afin que les programmes visant à renforcer la résilience se concentrent spécifiquement sur eux. 
- Analyse comment les différents acteurs participent aux différents systèmes et quels sont leurs relations de pouvoir afin de savoir où et comment renforcer le plus efficacement la résilience.

- Identifie les composants résistants du système, afin de comprendre comment opèrent les personnes déjà résilientes, et afin d'identifier des mesures qui permettent de mieux faire face à des risques inconnus ayant des effets inconnus. Cela permet de compléter les mesures actuelles de gestion des catastrophes, focalisées sur la capacité d'absorption avec des mesures soutenant les capacités d'adaptation et de transformation des individus et des sociétés.

Pour renforcer la résilience au sein de nos populations de l'Est du Congo, il faut au préalable comprendre les différents paradigmes liés aux risques, et cela pour chaque contexte, et saisir dans quelle couche de la société chaque risque peut-il être mieux géré. Ensuite il faut travailler à renforcer deux types de capacités pour mieux gérer ces risques et l'incertitude future (UNICEF, OECD, 2014), il s'agit de :

\section{- la capacité d'adaptation ;}

- la capacité de transformation.

Le renforcement de la résilience implique une approche systémique du management des risques, du changement et de l'incertitude. Un système peut être une unité de la société telle que l'individu, le foyer, un groupe de personnes ayant des caractéristiques communes, une communauté, une nation, une entité physique comme une infrastructure urbaine ou un écosystème.

Ce qui explique notre figure 2 , en ce qui concerne les étapes de l'analyse systémique de la résilience appliquée à l'Est du Congo.

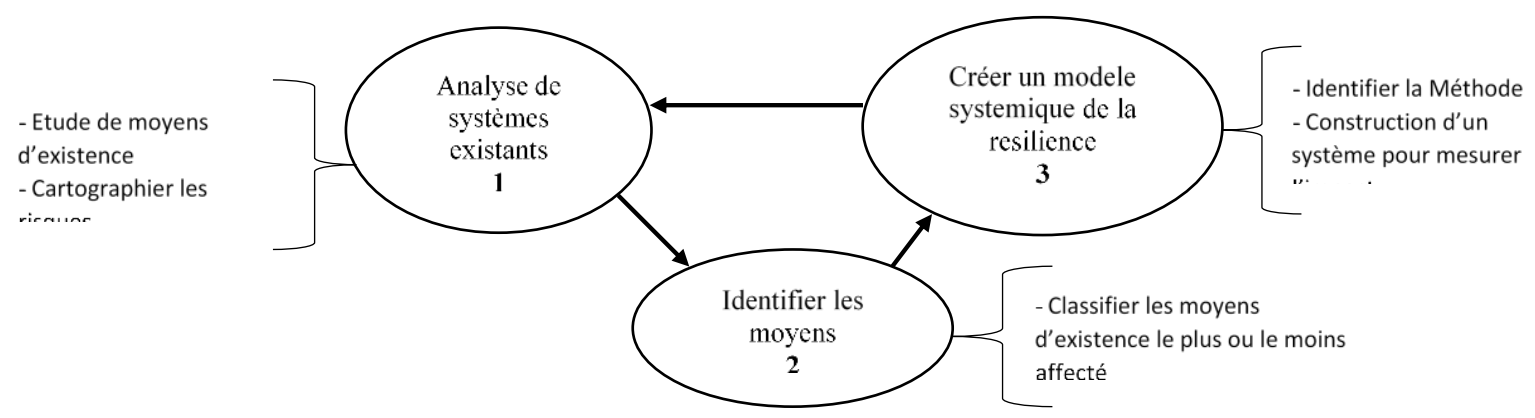

Figure 2. : Les étapes d'une analyse systémique de la résilience

\section{Situation sécuritaire à l'Est de la RD Congo : Facteur d'instabilité}

De 1996 à 2003, la République Démocratique du Congo a subi deux guerres au cours desquelles neuf armées étrangères et environ trente groupes armés se sont affrontés sur son territoire. Les guerres ont donné lieu à la partition temporaire du pays et couté la vie à des millions de personnes (Baaz \& Stern, 2010).

Dix ans plus tard, malgré la signature de plusieurs accords de paix, l'organisation de trois

élections présidentielles (en 2006, 2011 et 2018) et un investissement important des partenaires internationaux, l'Est de la RDC continue à abriter un grand nombre de factions armées et reste le thêâtre de cycles réguliers de violence. La complexité et la longévité du conflit congolais doivent être replacées et analysées dans le contexte d'une désintégration profonde de la société congolaise au cours des dernières décennies et de l'effet cumulé de deux cycles de conflits se renforçant mutuellement l'un régional, l'autre local aux dimensions locales, nationales, et régionales (Unité de stabilisation du DFID).

Les conflits à l'Est de la RDC peuvent être expliqués de la façon suivante. Dans un système social, l'aptitude à satisfaire ses besoins essentiels dépend des réseaux auxquels chacun appartient et à la 
position relative détenue dans le système. Pour pouvoir exploiter les institutions, ces dernières sont délibérément maintenues dans un état de faiblesse (International Alert, 2010).

C'est pourquoi :

- L'État congolais est particulièrement susceptible d'être victime d'ingérences extérieures ;

- Les Forces Armées de la République Démocratique du Congo et autres institutions étatiques sécuritaires ne disposent pas de capacités suffisantes afin de remplir leurs fonctions régaliennes auprès des populations civiles et sont parfois utilisées afin de poursuivre des intérêts privés ; et

- L'État n'est pas en mesure de fournir un cadre administratif et réglementaire répondant aux besoins de la population.

Compte tenu de ces trois dynamiques, la population vit dans une insécurité matérielle et institutionnelle permanente. Cela contribue à la formation de groupes exclusifs (en opposition à «l'autre »), souvent à caractère ethnique, desquels les individus dépendent pour leur sécurité. Ces tensions sont ensuite instrumentalisées par des entrepreneurs de conflit provoquant des cycles de violence (Peterman \& Palermo, 2011).

Par rapport à cette situation, la figure 3 nous démontre quatre dynamiques négatives principales qui se dégagent :

1. Dilemmes sécuritaires : Les dilemmes sécuritaires sont le produit de la nature patrimoniale de l'État, provoquant la faiblesse et la fragmentation des institutions de défense et de sécurité. L'État ne pouvant ou ne voulant pas protéger ses populations et leurs moyens de subsistance, les communautés ont tendance à se reposer sur les groupes armés pour leur défense.

2. Mobilisation autour de la terre et de l'identité : Les moyens de subsistance étant très limités, les communautés se replient sur des identités tribales exclusives, sources de solidarité et de ressources, et s'identifient en opposition à d'autres communautés. Le lien entre la terre et l'identité tribale ajoute une dimension économique à ce processus et solidifie encore plus les identités exclusives.

3. Exploitation des ressources naturelles: La disponibilité de précieuses ressources naturelles à forte valeur ajoutée (l'exploitation requiert de faibles apports financiers et utilise une main-d'œuvre à bas prix), est également attrayante pour les groupes armés.

4. Les dynamiques régionales: La porosité et la longueur des frontières avec les Etats voisins, les décennies - voire siècles - de flux de populations incontrôlés et de migrations saisonnières, et l'attrait des vastes ressources naturelles, ont contribué à la formation d'intérêts privés et publiques régionaux pour l'Est du Congo. Que ces intérêts soient légitimes ou pas, à présent, des individus, réseaux criminels et acteurs étatiques soutiennent des réseaux complexes qui contribuent à maintenir le conflit, soit directement, soit par procuration, soit parfois les deux à la fois.

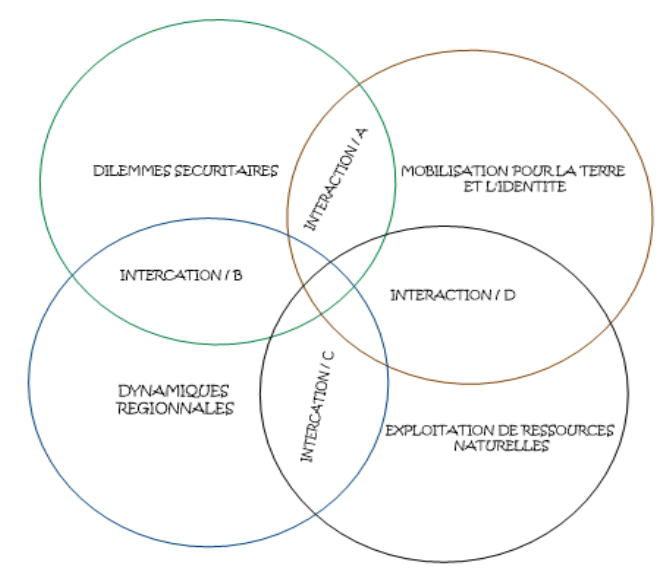

Figure $3:$ Interaction de quatre dynamiques négatives 


\section{INTERPRÉTATION DU MODÈLE SYSTÉMIQUE DE LA RÉSILIENCE APPLIQUÉE À L'EST DU CONGO}

Une lecture systémique des trajectoires de la commune Ungersheim présenté par Guy Koninckx (2018) dans son article intitulé : «Les parcours de transition d'Ungersheim : Être aspiré par le chaos des crises ou emprunter des trajectoires de résiliences » publié dans la revue de l'Union Européenne de Systémique, Acta Europeana Systemica $n^{\circ} 8$ nous a permis de proposer un modèle systémique de la résilience présenté dans la figure 4.

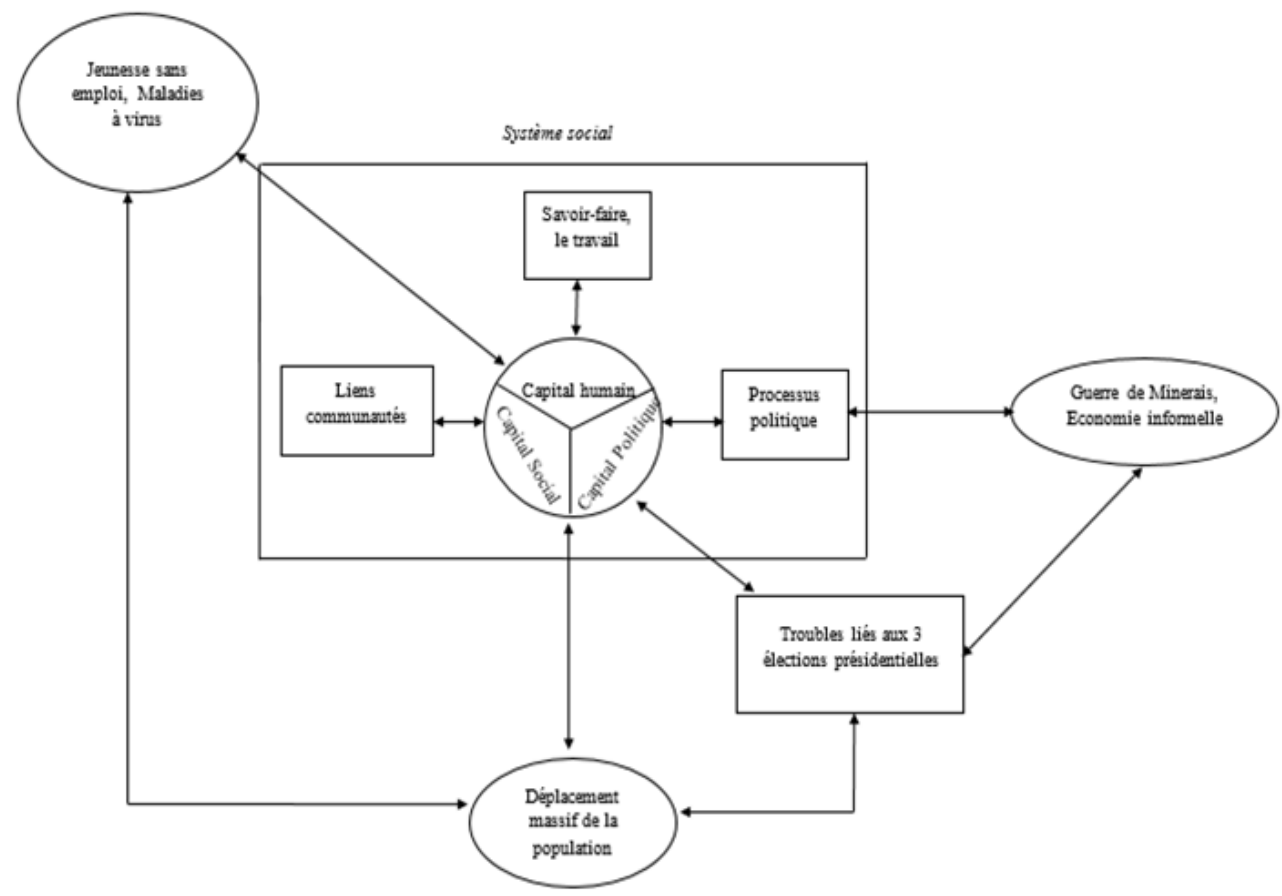

Figure 4 : Modèle systémique de la résilience

Notre modèle s'explique en ces termes, $\mathrm{Vu}$ que le capital humain, social et politique reste la clé majeure pour le développement d'une communauté ou d'une nation; Ainsi la persistance des différents conflits occasionne un changement dans les motivations des belligérants. Les aspects économiques prennent le pas sur les motivations politiques. Les contrôles sur les mines et les autres ressources naturelles deviennent la raison principale expliquant les changements d'alliances et les affrontements entre les groupes armés. Le conflit s'autofinance avec l'économie de guerre : Les ressources minières, le Coltan, Gaz Méthane et Autres.

La possibilité d'exploiter certaines de ces ressources avec un très faible investissement de départ et une haute intensité de main d'œuvre est une aubaine pour les groupes armés, qui trouvent des recrues parmi une population jeune et sans perspective de ressource. L'économie informelle de subsistance des ménages est parfois complétée par la contribution à l'économie de l'ombre, telle l'exploitation minière illégale comme stratégie de diversification de moyens d'existence ${ }^{1}$.

L'insécurité liés aux élections ainsi limitant l'accès à la prévention et au traitement de certaines maladies, institutions faibles ne permettant pas un accès au système de santé dans les zones reculées, déplacements de population, violences sexuelles et liées au genre, mais aussi la propagation rapide de maladies touche particulièrement l'Est du Congo, car le système sanitaire peine dans la plupart des cas à prévenir ou répondre à ces épidémies. De nombreux malades souffrent et décèdent de maladies évitables ou que l'on sait soigner. Ce coin du Pays est particulièrement affecté par la maladie à virus Ebola, le choléra, et les épidémies de rougeole reviennent de manière récurrente malgré les campagnes de vaccinations (Woods, 2006).

\footnotetext{
${ }^{1} \mathrm{http} / /$ revue-sesame-inra.fr/transition-reperes-pour-reussir-vers-une-approche-systemique-2-4/
} 
En plus des conséquences liées aux conflits eux-mêmes, tels les déplacement massifs, les violences et l'impact sur les moyens de subsistance des ménages, il faut aussi considérer les répercussions d'une exploitation non régulée des ressources en terme de dégradation de l'environnement et de la biodiversité, de pollution et de santé.

Ainsi la capacité d'un système social à s'adapter à ses perturbations que ça soit internes ou externes dépend de deux choses : d'une part de l'importance de l'aléa envisagé, car il est évident que si l'aléa envisagé conduit à la destruction du système, toute stratégie de résilience est vaine, et, d'autre part, elle dépend de la stratégie mise en place afin d'anticiper l'impact d'un choc. Une société qui connait les risques pesant sur elle, qui en connait par la même les conséquences et qui tire la leçon des perturbations passées, sera mieux à même de se redresser qu'une société n'ayant pas envisagé une telle hypothèse. Si, durant ces derniers siècles, la capacité d'un système social à résister à des perturbations s'illustrait par le patriotisme (capacité d'une nation à s'unir suite à un choc), aujourd'hui, les Etats doivent trouver d'autres moyens pour assurer leur pérennité d'où l'intérêt croissant des politiques pour ces questions de résilience (Holling, 1986).

\section{CONCLUSION}

La résilience s'intéresse fondamentalement à la capacité d'un système à supporter une perturbation, voire à l'intégrer dans son fonctionnement. Étudier la résilience d'un système social amène à focaliser le travail sur les moments critiques de changements dans la dynamique du système. Pour qu'une nation, un Etat et plus généralement un système social soit résilient c'est-à-dire qu'il puisse s'adapter à des perturbations internes et externes, naturelles ou humaines, il faut que soit mise en place une stratégie de résilience.

Une stratégie fondée sur la résistance concentre les moyens dans la prévention alors qu'une stratégie fondée sur l'acceptation du risque concentre les moyens sur le rétablissement de la situation et sur la limitation des dégâts et des conséquences. Une véritable stratégie de résilience vise, non pas à s'opposer à l'aléa comme cela peut être fait dans certains domaines, mais vise à en limiter les effets. Dès lors se pose la question de ce qui conditionne l'adaptabilité d'un système social :

- Qu'est-ce qui permet à un système social de se remettre plus ou moins facilement d'une perturbation plus ou moins importante ?

André Dauphiné et Damienne Provilo (2007), dans leur article La résilience pour un concept de gestion des risques, ont souligné le fait que, quel que soit le domaine d'application de la résilience, aucune étude n'a, à ce jour, identifié tous les facteurs qui influent la résilience d'un système. Néanmoins trois facteurs ont été identifiés comme améliorant la résilience d'une société à savoir :

- la diversité,

- la capacité d'auto-organisation du système et

- l'apprentissage.

Ainsi, un système disposant d'une multiplicité de valeurs et d'acteurs, disposant d'une capacité d'auto-organisation et d'un dispositif d'apprentissage (dans le sens d'une capacité d'apprendre des accidents passés pour s'adapter au futur) sera nécessairement plus résiliente. A l'inverse, une société hostile à l'innovation et à l'apprentissage, concentrant la prise de décision en un seul acteur entravant la prise d'initiative, serait, de fait, moins résiliente.

D'un point de vue théorique, la résilience s'insère dans le référentiel des systèmes complexes loin de l'équilibre : un système perturbé mais caractérisé par un comportement résilient parvient à se maintenir parce qu'il change. Ce point de vue est l'inverse d'une conception traditionnelle des systèmes en équilibre qui suppose qu'après une perturbation le système reviendra toujours vers le même état d'équilibre. De même manière, le cadre théorique de la résilience nécessite de relativiser certains critères qui, généralement, sont considérés comme positifs pour la reproduction d'un système. C'est le cas notamment pour l'adaptabilité et la stabilité de la trajectoire d'un système, qui peuvent avoir des effets négatifs sur la résilience. 


\section{RÉFÉRENCES}

BOURG, D. \& PAPAUX, A. (2015). Dictionnaire de la pensée écologique, Ed. Presses Universitaires de France, Paris.

BAAZ. \& STERN. (2010). La complexité de la violence : une analyse critique de les violences sexuelles en RDC. Document de travail du SIDA sur la violence sexiste.

CHABOT, P. (2015). L'âge des transitions, Ed. Presses Universitaires de France, Paris, p.68.

DAUPHINÉ, A. \& PROVITOLO, D. (2007). La résilience : un concept pour la gestion des risques. Annales de géographie, 2007/2 n ${ }^{\circ}$ 654, p. 115-125.

HOLLING, C.S. (1973). Resilience and stability of ecological systems. Annual review of ecology and systematics, $\mathrm{n}^{\circ} 4$, p. 1-23.

HOLLING, C.S. (1986). The resilience of terrestrial ecosystems: local surprise and global change. In Clark W.C., Munn R.E., dir., Sustainable development of the biosphere, Cambridge: Cambridge University Press, p. 192-217.

IVINZA LEPAPA, A. C. (2019). NFO : Nouvelles formes d'Organisation, Entreprises numérique, virtuelle, réseau et télétravail. Ed. Bookelis, Paris, p.20.

JACQUEMOT, P. (2009), «Ressources minérales, armes et violences dans les Kivus (RDC) », Hérodote, n 134, vol. 3, pp. 38-62.

KONINCKX, G. (2018). Les parcours de transition d'Ungersheim : être aspiré par le chaos des crises ou emprunter des trajectoires de résiliences, in Revue UES, Acta Europeana Systemica, n ${ }^{\circ} 08$, p. $307-$ 312.

MUTINGA MUTUISHAYI, M. (2001), La réconciliation nationale en RDC, Kinshasa, Ed. Média pour la Paix.

MUTINGA MUTUISHAYI, M. (2001), La problématique de la gouvernance en RDC: défis, Kinshasa, Medias pour la Paix.

PETERMAN, A. et PALERMO, T. (2011) «Estimations et déterminants de les violences sexuelles dont les femmes sont victimes en République démocratique du Congo ». American Journal of Public Health. Vol 101, No. 6.

UNICEF, OECD. (2014). Atelier sur l'analyse systémique de la résilience appliquée à l'Est de la République Démocratique du Congo, Goma, 29 et 30 avril 2014.

WOODS, D. D., (2006). Essential Characteristics of Resilience, in Hollnagel E., Woods D., Leveson N., Resilience Engineering: Concepts and Precepts, Ashgate.

WALLISER, B. (1977). Systèmes et modèles, introduction critique à l'analyse des systèmes. Ed. Seuil, Paris.

YACOV, H. (1998). Risk modeling, Assessment and Management, Wiley, New York.

Unité de stabilisation du DFID. Note sur les problématiques de la stabilisation en matière de genre. Sur Internet

http://www.stabilisationunit.gov.uk/attachments/article/520/SIN\%20Gender\%20011010.pdf

International Alert. (2010). "La guerre n'est pas encore finie : les perceptions communautaires des violences sexuelles et de ses racines en RDC. » Londres, Royaume-Uni.

$\mathrm{http} / / /$ revue-sesame-inra.fr/transition-reperes-pour-reussir-vers-une-approche-systemique-2-4/. 